\title{
SYSTEMS of TWO HEAVY QUARKS with EFFECTIVE FIELD THEORIES
}

\author{
N. BRAMBILLA \\ Dipartimento di Fisica dell'Università di Milano and INFN \\ Milano, Italy \\ *E-mail: nora.brambilla@mi.infn.it
}

\begin{abstract}
I discuss results and applications of QCD nonrelativistic effective field theories for systems with two heavy quarks.

Keywords: QCD, Effective Field Theories, Heavy Quarks
\end{abstract}

\section{Introduction}

Systems made by to heavy quarks play an important role in several high energy experiments. The diversity, quantity and accuracy of the data currently being collected is impressive and includes: data on quarkonium formation from BES at BEPC, E835 at Fermilab, KEDR (upgraded) at VEPP-4M, and CLEO-III, CLEO-c; clean samples of charmonia produced in B-decays, in photon-photon fusion and in initial state radiation, from the B-meson factory experiments, BaBar at SLAC and Belle at KEK, including the unexpected observation of large amounts of associated $(c \bar{c})(c \bar{c})$ production; the CDF and D0 experiments at Fermilab measuring heavy quarkonia production from gluon-gluon fusion in $p \bar{p}$ annihilations at $2 \mathrm{TeV}$; the Selex experiment at Fermilab with the preliminary observation of possible candidates for doubly charmed baryons; ZEUS and H1, at DESY, studying charmonia production in photon-gluon fusion; PHENIX and STAR, at RHIC, and NA60, at CERN, studying charmonia production, and suppression, in heavy-ion collisions. In the near future, even larger data samples are expected from the BES-III upgraded experiment, while the B factories and the Fermilab Tevatron will continue to supply valuable data for several years. Later on, new facilities will become operational (LHC at CERN, Panda at GSI, hopefully a Super-B factory, a Linear Collider, etc.) offering fantastic challenges and opportunities in this field. A comprehensive review of the experimental and theoretical status of heavy quarkonium physics 
may be found in the Cern Yellow Report prepared by the Quarkonium Working Group ${ }^{1}$. See also the talks at the last QWG meeting at BNL (cf. http://www.qwg.to.infn.it/).

On the theory side, systems made by two heavy quarks are a rather unique laboratory. They are characterized by the existence of a hierarchy of energy scales in correspondence of which one can define a hierarchy of nonrelativistic effective field theries (EFT), each EFT has less degrees of freedom left dynamical and is simpler. Some of these physical scales are large and may be treated in perturbation theory. The occurrence of these two facts makes two heavy quark systems accessible in QCD. In particular the factorization of high and low energy scales realized in the EFTs allows us to study low energy QCD effects in a systematic and under control way. Ultimately the simplest EFT defined at the ultrasoft energy, pNRQCD, allows us to study the Quantum Mechanics of a non-Abelian field theory.

\section{Scales and EFTs}

The description of hadrons containing two heavy quarks is a rather challenging problem, which adds to the complications of the bound state in field theory those coming from a nonperturbative QCD low-energy dynamics. A simplification is provided by the nonrelativistic nature suggested by the large mass of the heavy quarks and manifest in the spectrum pattern. Systems made by two heavy quarks are thus characterized by three energy scales, hierarchically ordered by the quark velocity $v \ll 1$ : the mass $m$ (hard scale), the momentum transfer $m v$ (soft scale), which is proportional to the inverse of the typical size of the system $r$, and the binding energy $m v^{2}$ (ultrasoft scale), which is proportional to the inverse of the typical time of the system. In bottomonium $v^{2} \sim 0.1$, in charmonium $v^{2} \sim 0.3$. In perturbation theory $v \sim \alpha_{\mathrm{s}}$. Feynman diagrams will get contributions from all momentum regions associated with these scales. Since these momentum regions depend on $\alpha_{\mathrm{s}}$, each Feynman diagram contributes to a given observable with a series in $\alpha_{\mathrm{s}}$ and a non trivial counting. For energy scales close to $\Lambda_{\mathrm{QCD}}$, the scale at which nonperturbative effects become dominant, perturbation theory breaks down and one has to rely on nonperturbative methods. Regardless of this, the non-relativistic hierarchy $m \gg m v \gg m v^{2}$ will persist also below the $\Lambda_{\mathrm{QCD}}$ threshold.

The wide span of energy scales involved makes also a lattice calculation in full QCD extremely challenging. We may, however, take advantage of the existence of a hierarchy of scales by substituting QCD with simpler but equivalent EFTs. A hierarchy of EFTs may be constructed by systemati- 
cally integrating out modes associated to energy scales not relevant for the two quark system. Such integration is made in a matching procedure that enforces the equivalence between QCD and the EFT at a given order of the expansion in $v$ and achieves a factorization between the high energy and the low energy contributions. By integrating out the hard modes one obtains Nonrelativistic QCD. In such EFT soft and ultrasoft scales are left dynamical and still their entanglement complicates calculation and power counting. We will focus here on the simplest EFT you can write down for two heavy quark systems, where only ultrasoft degrees of freedom remain dynamical. This is potential NRQCD ${ }^{\text {a }}$.

\section{3. $\mathrm{pNRQCD}$}

pNRQCD ${ }^{5-7}$ is the EFT for two heavy quark systems that follows from NRQCD by integrating out the soft scale. Here the role of the potential and the quantum mechanical nature of the problem are realized in the fact that the Schrödinger equation appears as zero problem for two quark states. We may distinguish two situations: 1) weakly coupled pNRQCD when $m v \gg \Lambda_{\mathrm{QCD}}$, where the matching from NRQCD to pNRQCD may be performed in perturbation theory; 2) strongly coupled pNRQCD when $m v \sim \Lambda_{\mathrm{QCD}}$, where the matching has to be nonperturbative. Recalling that $r^{-1} \sim m v$, these two situations correspond to systems with inverse typical radius smaller than or of the same order as $\Lambda_{\mathrm{QCD}}$.

\subsection{Weakly coupled pNRQCD}

The effective degrees of freedom are: low energy $Q \bar{Q}$ states (that can be decomposed into a singlet and an octet field under colour transformations) with energy of order $\Lambda_{\mathrm{QCD}}, m v^{2}$ and momentum $\mathbf{p}$ of order $m v$, plus ultrasoft gluons with energy and momentum of order $\Lambda_{\mathrm{QCD}}, m v^{2}$. All the gluon fields are multipole expanded (i.e. expanded in $r$ ). The Lagrangian is then given by terms of the type

$$
\frac{c_{k}(m, \mu)}{m^{k}} \times V_{n}\left(r \mu^{\prime}, r \mu\right) \times O_{n}\left(\mu^{\prime}, m v^{2}, \Lambda_{\mathrm{QCD}}\right) r^{n} .
$$

where the potential matching coefficients $V_{n}$ encode the non-analytic behaviour in $r$. At leading order in the multipole expansion, the singlet sector of the Lagrangian gives rise to equations of motion of the Schrödinger

a for an alternative and equivalent $\mathrm{EFT}$ (in the case in which $\Lambda_{\mathrm{QCD}}$ is the smallest scale) see ${ }^{4}$. 
type. Each term in the pNRQCD Lagrangian has a definite power counting; retardation (or non-potential) effects start at the NLO in the multipole expansion and are systematically encoded in the theory, they are typically related to nonperturbative effects ${ }^{6,7}$. Resummation of large logs (typically logs of the ratio of energy and momentum scales) can be obtained using the renormalization group ( $\mathrm{RG}$ ) adapted to the case of correlated scales 12; Poincaré invariance is not lost, but shows up in some exact relations among the matching coefficients ${ }^{9}$. The renormalon subtraction may be implemented systematically. ${ }^{7}$

\section{Applications of weakly coupled pNRQCD}

QCD Singlet Static potential. The singlet and octet potentials are well defined matching coefficients to be calculated in the perturbative matching. In ${ }^{11}$ a determination of the singlet potential at three loops leading log has been obtained and correspondingly also a determination of $\alpha_{V}$ showing how this quantity starts to depend on the infrared behaviour of the theory at three loops. The perturbative calculation of the static potential at (almost) three loops and with the RG improvement has been compared to the lattice calculation of the potential and found in good agreement up to about 0.25 $\mathrm{fm}{ }^{17}$.

$b$ and $c$ masses.

Heavy quarkonium is one of the most suitable system to extract a precise determination of the mass of the heavy quarks $b$ and $c$. Perturbative determinations of the $\Upsilon(1 S)$ and $J / \psi$ masses have been used to extract the $b$ and $c$ masses. The main uncertainty in these determinations comes from nonperturbative contributions (local and nonlocal condensates ${ }^{6}$ ) together with possible effects due to subleading renormalons ${ }^{7}$. A recent analysis performed by the QWG ${ }^{1}$ and based on all the previous determinations indicates an error of about $50 \mathrm{MeV}$ both for the bottom (1\% error) and in the charm (4\% error) mass.

\section{Perturbative quarkonium spectrum.}

$B_{c}$ mass. Table 1 shows some recent determinations of the $B_{c}$ mass in perturbation theory at NNLO accuracy compared with a recent lattice study ${ }^{16}$ and the value of the CDF experimental $B_{c}$ mass. This would support the assumption that nonperturbative contributions to the quarkonium ground state are of the same magnitude as NNLO or even NNNLO corrections, which would be consistent with a $m v^{2} \gtrsim \Lambda_{\mathrm{QCD}}$ power counting. Hyperfine splittings. $c \bar{c}, b \bar{b}, B_{c}$ ground state hyperfine splittings have been recently calculated at NLL in ${ }^{19}$. The prediction for $\eta_{b}$ mass is $M\left(\eta_{b}\right)=9421 \pm 10(\mathrm{th})_{-8}^{+9}\left(\delta \alpha_{\mathrm{s}}\right) \mathrm{MeV}$. The logs resummation seems to be 
important. If the experimental error in future measurements of $M\left(\eta_{b}\right)$ will not exceed few Mev, the bottomonium hyperfine separation will become a competitive source of $\alpha_{s}\left(M_{Z}\right)$ with an estimated accuracy of \pm 0.003 .

Radiative transitions (M1). A theory of M1 transitions in heavy quarkonium has been recently formulated using pNRQCD ${ }^{20}$. This may shed some light on recent CLEO results on radiative M1 transitions in the $\eta_{b}$ search that have ruled out several models. No large anomalous quarkonium magnetic moment is generated.

Seminclusive radiative decays of $\Upsilon(1 S)$. In ${ }^{22}$ the end-point region of the photon spectrum in semi-inclusive radiative decays of heavy quarkonium has been discussed using Soft-Collinear Effective Theory and pNRQCD. Including the octet contributions a good understanding of the experimental data is obtained.

Gluelump spectrum. In pNRQCD ${ }^{6,21}$ the full structure of the gluelump spectrum has been studied, obtaining model independent predictions on the shape, the pattern, the degeneracy and the multiplet structure of the hybrid static energies for small $Q \bar{Q}$ distances that well match and interpret the existing lattice data.

Properties of baryons made of two or three heavy quarks. Recently the SELEX experiment has detected first signals from three-body bound states made of two heavy quarks and a light one. The two heavy quark part of such systems may be treated in pNRQCD and a precise predication for the hyperfine interaction may be obtained ${ }^{23}$.

\begin{tabular}{|c|cccc|}
\hline \multicolumn{5}{|c|}{$B_{c}$ mass $(\mathrm{MeV})$} \\
\hline${ }^{18}$ (expt) & ${ }^{16}$ (lattice) & ${ }^{15}(\mathrm{NNLO})$ & ${ }^{13}(\mathrm{NNLO})$ & ${ }^{14}(\mathrm{NNLO})$ \\
\hline $6287 \pm 4.8 \pm 1.1$ & $6304 \pm 12_{-0}^{+12}$ & $6326(29)$ & $6324(22)$ & $6307(17)$ \\
\hline
\end{tabular}

\subsection{Strongly coupled $p N R Q C D$}

In this case the matching to pNRQCD is nonperturbative ${ }^{25}$. In the situation where the other degrees of freedom (like those associated with heavylight meson pair threshold production and heavy hybrids) develop a mass gap of order $\Lambda_{\mathrm{QCD}}$, the quarkonium singlet field $\mathrm{S}$ remains as the only low energy dynamical degree of freedom in the pNRQCD Lagrangian (if no ultrasoft pions are considered), which reads ${ }^{25,26,6,7}$ :

$$
L_{\mathrm{pNRQCD}}=\operatorname{Tr}\left\{\mathrm{S}^{\dagger}\left(i \partial_{0}-\frac{\mathbf{p}^{2}}{2 m}-V_{S}(r)\right) \mathrm{S}\right\} .
$$

In this regime we recover the quark potential singlet model from pNRQCD. The matching potential $V_{S}$ (static and relativistic corrections) is nonper- 
turbative: the real part controls the spectrum and the imaginary part controls the inclusive decays. The potential is calculated in the nonperturbative matching procedure between NRQCD and pNRQCD ${ }^{25,7}$. Advantages of this approach include: factorization of hard (in the NRQCD matching coefficients) and soft scales (contained in Wilson loops or nonlocal gluon correlators); the low energy objects being only glue dependent, confinement investigations, on the lattice and in $\mathrm{QCD}$ vacuum models become feasible ${ }^{24}$; the existence of a power counting indicating leading and subleading terms in quantum-mechanical perturbation theory; the quantum mechanical divergences (like the ones coming from iterations of spin delta potentials) are absorbed by NRQCD matching coefficients. The potentials evaluated on the lattice once used in the Schrödinger equation produce the spectrum. The calculations involve only QCD parameters (at some scale and in some scheme).

\section{Applications of strongly coupled pNRQCD}

Nonperturbative potentials and Spectrum. Recently the multilevel algorithm has been applied to the lattice evaluation of field strength insertion inside the Wilson loop average, producing very precise data for the spin dependent potentials and a first evaluation of the nonperturbative potential at order $1 / m^{27}$. This is the first step towards a precise determination of the nonperturbative matching potentials on the lattice.

Decays. The inclusive quarkonium decay widths in pNRQCD can be factorized with respect to the wave function (or its derivatives) calculated in zero, which is suggestive of the early potential models results: $\Gamma(\mathrm{H} \rightarrow \mathrm{LH})=F\left(\alpha_{\mathrm{s}}, \Lambda_{\mathrm{QCD}}\right) \cdot|\psi(0)|^{2}$. Similar expressions hold for the electromagnetic decays. However, the coefficient $F$ depends here both on $\alpha_{\mathrm{s}}$ and $\Lambda_{\mathrm{QCD}}$. In particular all NRQCD matrix elements, including the octet ones, can be expressed through pNRQCD as products of universal nonperturbative factors by the squares of the quarkonium wave functions (or derivatives of it) at the origin. The nonperturbative factors are typically integral of nonlocal electric or magnetic correlators and thus depends on the glue but not on the quarkonium state ${ }^{26}$. Typically $F$ contains both the NRQCD matching coefficients at the hard scale $m$ and the nonperturbative correlators at the low energy scale $\Lambda_{\mathrm{QCD}}$. The nonperturbative correlators, being state independent, are in a smaller number than the nonperturbative NRQCD matrix elements and thus the predictive power is increased in going from NRQCD to pNRQCD. Thus, having fixed the nonperturbative parameters on charmonium decays, new model-independent QCD predictions can be obtained for the bottomonium decay widths ${ }^{26}$. 


\section{References}

1. N. Brambilla et al., CERN Yellow Report, CERN-2005-005, arXiv:hep$\mathrm{ph} / 0412158$.

2. W. E. Caswell and G. P. Lepage, Phys. Lett. B 167 (1986) 437.

3. G. T. Bodwin, E. Braaten and G. P. Lepage, Phys. Rev. D 51 (1995) 1125

4. A. V. Manohar and I. W. Stewart, Phys. Rev. D 62, 014033 (2000); A. H. Hoang and I. W. Stewart, Phys. Rev. D 67, 114020 (2003).

5. A. Pineda and J. Soto, Nucl. Phys. Proc. Suppl. 64, 428 (1998);

6. N. Brambilla, A. Pineda, J. Soto and A. Vairo, Nucl. Phys. B 566, 275 (2000).

7. N. Brambilla, A. Pineda, J. Soto and A. Vairo, Rev. Mod. Phys. 77, 1423 (2005)

8. A. Gray et al. arXiv:hep-lat/0507013.

9. N. Brambilla, D. Gromes and A. Vairo, Phys. Lett. B 576, 314 (2003); Phys. Rev. D 64, 076010 (2001);

10. N. Brambilla, A. Pineda, J. Soto and A. Vairo, Phys. Lett. B470, 215 (1999); B. A. Kniehl and A. A. Penin, Nucl. Phys. B 563, 200 (1999).

11. N. Brambilla et al., Phys. Rev. D 60, 091502 (1999).

12. A. Pineda, Phys. Rev. D 65, 074007 (2002); A. Pineda and J. Soto, Phys. Lett. B 495, 323 (2000).

13. N. Brambilla, Y. Sumino and A. Vairo, Phys. Lett. B 513, 381 (2001).

14. N. Brambilla, Y. Sumino and A. Vairo, Phys. Rev. D 65, 034001 (2002).

15. N. Brambilla and A. Vairo, Phys. Rev. D 62, 094019 (2000).

16. I. F. Allison et al. arXiv:hep-lat/0411027.

17. A. Pineda, J. Phys. G 29, 371 (2003); Y. Sumino, arXiv:hep-ph/0505034.

18. D. Acosta et al. [CDF Collaboration], arXiv:hep-ex/0505076.

19. B. A. Kniehl, A. A. Penin, A. Pineda, V. A. Smirnov and M. Steinhauser, Phys. Rev. Lett. 92, 242001 (2004); A. A. Penin, A. Pineda, V. A. Smirnov and M. Steinhauser, Phys. Lett. B 593, 124 (2004).

20. N. Brambilla, Y. Jia and A. Vairo, Phys. Rev. D 73, 054005 (2006)

21. G. S. Bali and A. Pineda, Phys. Rev. D 69, 094001 (2004).

22. X. Garcia i Tormo and J. Soto, Phys. Rev. D 72, 054014 (2005). Phys. Rev. Lett. 96, 111801 (2006)

23. N. Brambilla, A. Vairo and T. Rosch, Phys. Rev. D 72, 034021 (2005); S. Fleming and T. Mehen, arXiv:hep-ph/0509313.

24. N. Brambilla and A. Vairo, arXiv:hep-ph/9904330;

25. A. Pineda and A. Vairo, Phys. Rev. D 63, 054007 (2001) N. Brambilla et al. Phys. Rev. D 63, 014023 (2001); Phys. Lett. B 580, 60 (2004).

26. N. Brambilla, D. Eiras, A. Pineda, J. Soto and A. Vairo, Phys. Rev. D67, 034018 (2003); Phys. Rev. Lett. 88, 012003 (2002).

27. Y. Koma, M. Koma and H. Wittig, arXiv:hep-lat/0607009. PoS LAT2005, 216 (2006)

28. N. Brambilla, P. Consoli and G. M. Prosperi, Phys. Rev. D 50, 5878 (1994); 